\section{EPEC anti-phagocytosis}

Enteropathogenic Escherichia coli (EPEC), a major cause of severe infantile diarrhea, prevents its own uptake by macrophages. In the European Molecular Biology Organization Journal, Finlay and colleagues show that EPEC "anti-phagocytosis" occurs by prevention of the F-actin polymerization required for phagocytosis. Upon contact with the host cell, EPEC uses a type III secretion system to translocate the intimin receptor, Tir, into the host cell membrane. However, the authors show that EPEC anti-phagocytosis occurs via a TIR-independent process. EPEC blocks uptake by inhibiting a PI3K-mediated pathway and translocates effectors other than TIR to interfere with actin-driven host cell processes. Thus inhibition of a PI3K-mediated pathway enables a bacterial pathogen to avoid clearance by macrophages.

EM BO J. 20, 1245-1258 (2001)

\section{The complex business of CTLA-4}

An efficient immune response requires both an antigen-specific and costimulatory signal. The most important costimulatory signals are provided by CD28 and CTLA-4, which augment and attenuate the immune response, respectively. Given their central role in regulating $\mathrm{T}$ cell activity, these signaling pathways offer new targets for immunotherapy. Two papers published in Nature, by Schwartz et al. and Stamper et al., describe the crystal structure complex of human CTLA-4 and its ligand, B7-1. Both groups show that CTLA-4 and B7-1 can form homodimers. These pack together in the crystal lattice to form a regular arrangement in which the bivalent CTLA-4 homodimers bridge bivalent B7-1 homodimers. This "zipper-like" oligomerization or periodic organization provides the structural basis for formation of unusually stable signaling complexes

Nature 410, 604-608 \& 608-611 (2001)

\section{Microglia andT GF- $\beta 1$ in AD}

Alzheimer's disease (AD) is a progressive dementia characterized by amyloid plaques made up of amyloid peptide (A $\beta)$ in the brain. In Nature Medicine, Wyss-Coray and colleagues show that a modest increase in production of TGF- $\beta 1$ from astroglia in mice transgenic for human TGF- $\beta 1$ and amyloid resulted in reductions in the number of amyloid plaques, the overall $A \beta$ load and the number of damaged neurons. Further data suggests that this beneficial effect of TGF $\beta 1$ might be mediated by activated microglia; TGF $\beta 1$ increases microglial activation; the double transgenic mice had widespread microglial activation and this cytokine stimulated cultured microglia to clear $A \beta$.

Nature M ed. 7, 612-618 (2001)

\section{The common CD91 receptor}

Heat shock proteins (hsps) such as gp96 and hsp70 form complexes with antigenic peptides, which are then taken up by APCs and presented by MHC class I molecules. Recently, the $\alpha_{2}$ macroglobulin receptor CD91 was identified as the receptor for gp96. In Immunity, Srivastava and colleagues show that, despite a lack of structural homology, other hsps-such as calreticulin, hsp70 and hsp90-also use CD91. Primary macrophages, and mature and immature dendritic cells, were all able to re-present hsp-chaperoned peptides. Like hsp70-chaperoned peptides, gp96-chaperoned peptides follow the classical proteosome and TAP-dependent pathway of endogenous antigen presentation by MHC class I molecules. These results suggest that CD91 represents a common receptor for hsp-chaperoned peptides that use similar downstream pathways for re-presentation.

Immunity 14, 303-313 (2001)

\section{GCs attenuateTCR signals}

Glucocorticoids (GCs) affect peripheral immune responses by inhibiting $\mathrm{T}$ cell immunity and causing impaired cytokine production and effector function. In the Journal of Experimental Medicine, van Laethem et al. show that dexamethasone, a synthetic GC, inhibits the early signaling events initiated upon TCR ligation, including tyrosine phosphorylation of TCR-associated substrates such as the $\zeta$ chain, ZAP70 kinase and the transmembrane adaptor molecule for activation of T cells. Hypophosphorylation correlated with an altered membrane compartmentalization of these molecules. This work indicates that the nature of membrane- proximal signals delivered by the TCR is sensitive to the hormonal environment and suggests a new mechanism by which steroids may regulate $\mathrm{T}$ cell differentiation and responsiveness.

J. Exp. Med. 193, 471-481 (2001)

\section{Slit remains repulsive}

The chemokine family is important in leukocyte chemotaxis. In Nature, Wu et al. report that Slit, a secreted protein previously known for its repulsive role in axon guidance and neuronal migration, also inhibits leukocyte chemotaxis. They also showed that Robo, a Slit receptor expressed in the hematopoietic system, is involved in mediating Slit inhibitory responses in leukocyte chemotaxis. Robo contains a single transmembrane domain, thus showing that seven-transmembrane receptors are not the sole receptors mediating chemokine responses in leukocytes. These results reveal that the activity of a neuronal guidance cue plays a role in leukocyte chemotaxis and that this underlies a conserved guidance mechanism of cell migration. Slit may be a new therapeutic target for diseases involving leukocyte migration and chemotaxis.

Nature 410, 948-952 (2001)

\section{Natural killer rejection}

Adaptive $\mathrm{T}$ cell responses participate in self and nonself discrimination that mediates rejection of nonsyngeneic tissues. T cell activation and subsequent maturation to effector lymphocytes is CD28-dependent. However, in CD28-deficient mice, $\mathrm{T}$ cells can still mediate acute graft rejection. In Nature Medicine, Maier et al. help explain this intriguing conundrum. They show that only simultaneous inhibition of NK receptor-bearing cells and CD28 costimulation blockade could lead to long-term acceptance of allogeneic grafts. These results suggest that NK and/or NK T cells infiltrate the graft tissue, become activated due to the absence of silencing signals via self-MHC molecules and provide "help" to CD28-deficient T cells, thus overcoming costimulation deficiency. Inactivation of NK receptor-bearing cells may provide a potential new target for survival of solid organ transplants.

Nature Med. 7, 557-562 (2001) 